\title{
Efecto de las enterobacterias en pacientes con periodontitis crónica
}

\section{Effect of enterobacteriaceae in patients with chronic periodontitis}

\author{
ARDILA MEDINA CM*
}

Ardila Medina CM. Efecto de las enterobacterias en pacientes con periodontitis crónica. Av Periodon Implantol. 2010; 22, 1:27-35

\section{RESUMEN}

La periodontitis crónica es una enfermedad infecciosa asociada a microorganismos gram-negativos anaerobios. Esta entidad de naturaleza crónica y de alta prevalencia en la población, ha sido relacionada con un riesgo aumentado a infarto agudo del miocardio, accidente cerebrovascular, bajo peso al nacer y parto pretérmino.

Estudios microbiológicos en pacientes con periodontitis en diferentes partes del mundo han mostrado altas prevalencias de sobreinfección por enterobacterias, las cuales podrían complicar el cuadro clínico de los pacientes y la respuesta a la terapia periodontal. Así mismo, el lipopolisacárido presente en su pared, ha demostrado estimular la activación celular y la producción de citoquinas proinflamatorias que podrían aumentar el riesgo de enfermedades sistémicas en estos pacientes. Aunque la terapia periodontal reduce los marcadores inflamatorios en suero y ha mostrado una reducción en la incidencia de bajo peso al nacer y parto pretérmino, no se ha estudiado el efecto de las enterobacterias a nivel subgingival sobre la respuesta a la terapia básica y los efectos sistémicos que pueda desencadenar. El objetivo de esta revisión es proporcionar fundamentos que permitan de alguna manera dilucidar tal problemática.

PALABRAS CLAVE: Periodontitis, microbiología, enterobacterias, patógenos.

\section{SUMMARY}

The chronic periodontitis is an infectious disease associated to anaerobic gram negative microorganisms. This organization of chronic nature and high prevalence in the population has been associated with a risk increased to acute infarct of the myocardium, stroke, low birth weight and preterm infants. Microbiological studies in patients with periodontitis in different parts from the world have shown high prevalences of sobreinfection by enterobacteriaceae, which could complicate the clinical picture of the patients and the answer to the periodontal therapy. Also, the present lipopolisacaric one in its wall, has demonstrated to stimulate the cellular activation and the production of proinflammatory cytokines that could increase the risk of systemic diseases in these patients. Although the periodontal therapy reduces the inflammatory serum markers and has shown a reduction in the incidence of low birth weight and preterm infants, has not studied the effect of enterobacteriaceae at subgingival level on the sistemic answer to the basic therapy and effects that can trigger. The objective of this revision is to provide foundations that somehow allow to explain so problematic.

KEY WORDS: Periodontitis, microbiology, enterobacteriaceae, pathogens.

Fecha de recepción: 18 de julio de 2008.

Fecha de aceptación: 21 de julio de 2008.

Profesor Asistente Universidad de Antioquia.

Presidente de la Sociedad Colombiana de Periodoncia-Regional Antioquia.

Candidato a PhD en Epidemiología. 


\section{INTRODUCCIÓN}

Entre las enfermedades más difundidas en el mundo se encuentran las periodontales que pueden afectar al $48 \%$ de la población adulta, prevalencia que varía según condiciones culturales, sociales, económicas y políticas (1). Dentro del grupo de las enfermedades periodontales se encuentra la periodontitis crónica, la cual es una patología infecciosa caracterizada por la formación de bolsas, que puede afectar un número variable de dientes. Además, ocasiona inflamación de los tejidos de soporte dental, pérdida ósea y de inserción (2). Las lesiones pueden ser tan graves que pueden ocasionar pérdida de los dientes y alteración de la función masticatoria y estética, afectando por consiguiente el bienestar y calidad de vida de las personas (3).

Como ya se mencionó, la periodontitis crónica es una enfermedad infecciosa y se ha documentado extensamente el papel de la microflora subgingival en su etiología $(4,5)$. Diferentes estudios han demostrado similitudes en los periodontopatógenos y han encontrado que en los pacientes con periodontitis crónica, están presentes y en forma conjunta, la mayoría de los siguientes microorganismos: Porphyromona gingivalis, Actinobacillus actinomycetemcomitans, Tannerella forsythensis, Eikenella corrodens, Campylobacter rectus, Peptoestreptococo micros, Treponema denticola y la Prevotella intermedius $(6,7)$. Sin embargo, algunas investigaciones han demostrado que la frecuencia relativa de cada microorganismo varía entre poblaciones de diferentes orígenes geográficos, concluyendo que la prevalencia de patógenos periodontales específicos cambia entre individuos del mismo ambiente y entre distintas etnias y países (8-13). Además de los ya mencionados, en los ambientes subgingivales de algunos pacientes con periodontitis crónica, se han encontrado otros microorganismos tales como las enterobacterias. No obstante, la frecuencia de este fenómeno también es diversa entre diferentes regiones del mundo $(9,14-19)$.

El componente microbiano de la bolsa periodontal parece modificar algunos aspectos clínicos de la periodontitis, entre ellos la respuesta al tratamiento (7, 20). El tratamiento en pacientes con periodontitis se realiza mediante terapia mecánica consistente en técnicas de raspado y alisado radicular, las cuales han mostrado efectividad en múltiples estudios en términos de mejoría de los parámetros clínicos y microbiológicos. Los parámetros para evaluar la respuesta clínica al tratamiento están relacionados con reducción en la profundidad de la bolsa, ganancia de inserción clínica, disminución de la inflamación gingival y el sangrado al sondaje; y los microbiológicos con reducción significativa de la mayoría de los patógenos periodontales a corto y largo plazo $(21,22)$.

Algunas investigaciones han reportado que no todos los pacientes, ni los sitios del diente responden uniforme y favorablemente a la terapia mecánica convencional, y que esto es explicado en parte por la composición microbiana de la placa subgingival. Slots (14), Listgarten (23) y Handal (24), encontraron en diferentes estudios, que en pacientes tratados con terapia mecánica tradicional no había mejoría en aquellas bolsas periodontales que presentaban enterobacterias.

En algunas regiones del mundo se necesitan protocolos adecuados para el tratamiento de la periodontitis crónica, debido a que la presencia de enterobacterias en placa subgingival le proporciona características microbiológicas particulares a tales poblaciones (8). Por consiguiente, es importante buscar terapéuticas basadas en las particularidades que tiene la enfermedad en algunos países, generando nuevo conocimiento en este sentido que contribuya al desarrollo de protocolos específicos. Además, el tratamiento efectivo de la periodontitis crónica tiene implicaciones en la salud pública debido a que esta enfermedad genera discapacidad y deterioro de la calidad de vida, no solo porque produce movilidad y pérdida de los dientes, sino también por su asociación con problemas sistémicos como aterosclerosis, enfermedad coronaria y enfermedades respiratorias, y complicaciones obstétricas como parto pretérmino y bajo peso al nacer $(25,26)$.

El objetivo de esta revisión es dilucidar el papel de las enterobacterias en la etiología de la periodontitis crónica de tal manera que proporcione información pertinente para la adecuación de pautas de tratamiento y aporte datos importantes que motiven a realizar estudios analíticos que permitan incorporar guías de atención integral basadas en la evidencia que no posee el actual esquema de prestación de servicios en algunos países.

\section{GENERALIDADES Y PATOGENIA DE LAS ENTEROBACTERIAS}

Las enterobacterias constituyen la familia enterobacteriaceae que comprende bacilos gram negativos aerobios y anaerobios facultativos. Su clasificación por géneros se muestra en la tabla 1. 


\section{TABLA 1.- CLASIFICACIÓN DE LAS ENTEROBACTERIAS POR GÉNERO}

\begin{tabular}{|l} 
Escherichia \\
Shigella
\end{tabular}

Escherichia

Shigella

Klebsiella

Enterobacter

Hafnia

Proteus

Morganella

Yersinia

Edwardsiella

Citrobacter

Erwinia

Microbiológicamente las enterobacterias se caracterizan por no formar esporas, crecen tanto en aerobiosis como en anaerobiosis, fermentan la glucosa, no producen oxidasa, y tienen una movilidad variable $(27,28)$. Son gramnegativos que poseen una membrana citoplasmática, una cubierta de peptidoglicano y una compleja pared celular que comprende la cápsula, la cual contiene lipopolisacáridos (LPS) y canales para la penetración de antibióticos y nutrientes $(27,28)$. El LPS de la pared celular comprende una zona más interna que contiene la molécula del lípido $A$ de la que depende la actividad biológica de la endotoxina, responsable de la producción del shock endotóxico característico de estas bacterias. En la parte más externa se encuentra el LPS que constituye el antígeno $O$, que está constituido por una serie de cadenas laterales repetidas de polisacáridos. Junto con otros factores, la presencia de antígeno $\mathrm{O}$ media la resistencia bacteriana al efecto bactericida del suero normal. Los aislados resistentes a la acción bactericida del suero sobreviven más tiempo en sangre y por ello causan infecciones hematógenas, diseminadas y más agresivas. Existen tres tipos de antígenos de superficie que sirven para serotipar e identificar a las enterobacterias: el antígeno somático o antígeno $\mathrm{O}$, el antígeno flagelar $\mathrm{o}$ antígeno $\mathrm{H}$, y el antígeno capsular o antígeno $\mathrm{K}(27,28)$.
En ciertas especies como en $E$. coli, la presencia de un determinado antígeno $\mathrm{O}, \mathrm{H}$ y $\mathrm{K}$ determina la patogenicidad de una cepa. Así, ciertos antígenos $O$ actúan como factores de adhesión/colonización necesarios para la producción de infección, otros actúan como factores de colonización y como toxinas $(27,28)$. Los antígenos $\mathrm{H}$ son proteínas encontradas en los flagelos de estas bacterias y están ligados a la producción del síndrome hemolítico urémico y podrían ser responsables de la capacidad de progresión de las enterobacterias a través de las vías urinarias. Por último, los antígenos $\mathrm{K}$ son polisacáridos ácidos situados en la superficie celular. Algunos de ellos como el antígeno capsular $\mathrm{Kl}$ de $E$. coli se asocian con el desarrollo de meningitis neonatal, bacteriemia e infección urinaria $(27,28)$. Otros factores de virulencia lo constituyen las fimbrias, que son prolongaciones filamentosas que permiten la adherencia de las bacterias a receptores específicos de las células mucosas y epiteliales de las vías respiratorias, digestivas y genitourinarias Tal adherencia es extraordinariamente selectiva. Así, las fimbrias del tipo I, que son muy comunes y se hallan en multitud de cepas de E. coli, Klebsiella spp. y Salmonella spp., se unen específicamente a receptores que contienen manosa. Otro factor de virulencia que contienen las enterobacterias son los plásmidos, fragmentos de ADN extracromosómico transmisibles de bacteria a bacteria, no siempre de la misma especie, que permiten transmitir la resistencia a antibióticos (plásmidos R) o la producción de toxinas $(27,28)$.

Se ha sugerido que la presencia de bacterias entéricas podría complicar el cuadro clínico de los pacientes con periodontitis, las cuales podrían llevar a complicaciones sistémicas al entrar en el torrente sanguíneo, induciendo septicemias en pacientes inmunosuprimidos. Sin embargo, el mayor impacto clínico podría estar relacionado con su capacidad de activar monocitos por parte de su potente LPS (29) y contribuir a su activación vía citoquinas aumentando el riesgo cardiovascular. De esta manera las enterobacterias en placa subgingival de pacientes con periodontitis aumentarían el riesgo de enfermedad cardiovascular, el bajo peso al nacer y el parto pretérmino.

La exposición al LPS de dichas bacterias es probablemente el mayor estimulo al sistema inmune y uno de los más intensos. El LPS además de estar asociado con shock séptico, produce exacerbación de procesos alérgicos, desviación de respuesta celular Th2 (anticuerpos) a fenotipo inflamatorio Thl, con base en la inducción de citoquinas. Además, a nivel celular el LPS y las lipoproteínas son iniciadores de mediadores inflama- 
torios, activación celular, proliferación, inducción e inhibición de apoptosis (30).

La acción del LPS de bacterias periodontopáticas difiere de la observada en bacterias entéricas y esto puede ser explicado por las diferencias estructurales y bioquímicas de este componente en las diferentes especies (31). Cuando el LPS de una bacteria gramnegativa es liberado dentro del plasma es opsonizado por una proteína ligadora específica (LBP) en el suero. La unión LPS-LBP puede iniciar una significante activación celular dentro del plasma especialmente en monocitos circulantes. El complejo LPS-LBP es reconocido por el CD14 de estas células las cuales se encuentran asociadas con la superficie celular (32). La unión de CD 14 con el complejo LPS-LBP dispara la asociación de las moléculas CD14 ocupadas con un receptor de señalización. Esto estimula al fagocito a sintetizar una serie de citoquinas como TNF, IL-1, IL-6, IL-10, IL-12 e IL-15 (33). El CD 14 transfiere el LPS a un receptor intramembranal para iniciar la traducción de señal (Toll like receptor TLRs). La familia TLR consiste de receptores expresados en las células involucradas en la respuesta inmune innata como son el monocito, el macrófago, la célula dendrítica y el polimorfonuclear, en la célula $T$ y la célula B (34). En el humano el primer TLR caracterizado fue el TLR4 al cual se le ha atribuido el reconocimiento del LPS (34). Tapping y colaboradores (35) compararon las citoquinas proinflamatorias derivadas de la activación del TLR2 con la activación del TLR4 estimulados en ambos casos con LPS de la enterobacterias $E$. coli y $S$. minnesota, mostrando que una vez bloqueado el TLR4 se evidencia una disminución significativa en la producción de citoquinas (TNFalfa e IL8) a diferencia de la producción en los casos en que fue bloqueado el TLR2. Este estudio sugiere que el TLR4 es un receptor de señalización restringido a los LPS derivados de la familia Enterobacteriaceae (35).

Numerosos estudios han comparado la producción de citoquinas por monocitos retados con LPS de $P$ gingivalis, con la producción de ellas por monocitos enfrentados con $E$ coli, mostrando resultados contradictorios. Mientras que Shapira (36) encontró que el LPS de $P$ gingivalis tiene la misma habilidad para la producción de citoquinas, Bainbridge y colaboradores (37) reportaron que $P$ gingivalis mostraba menor habilidad para la producción de citoquinas. Ogawa y Uchida (29) mostraron que el LPS de $P$ gingivalis presentaba menor habilidad para la producción de IL-lbeta y TNF alfa pero una habilidad similar para la producción de IL 6. Roberts y colaboradores (38) compararon el efecto de los LPS de $E$ coli y $P$ gingivalis en su capacidad de producción de IL-lbeta y TNF alfa encontrando que ambos tipos de LPS activan las citoquinas.

\section{LOCALIZACIÓN}

Gran parte de las enterobacterias son habitantes normales del aparato digestivo de humanos y animales y residen como microflora normal en ambientes naturales (39). Estos microorganismos han sido reportados como transeúntes de la microflora bucal, y pueden encontrarse sobre la superficie mucosa, dientes y en el área subgingival de pacientes con enfermedad periodontal avanzada (40). También se han aislado de la cavidad bucal de individuos que usan dentaduras y pueden estar asociados a halitosis (41). Se ha demostrado que el $10,2 \%$ de los pacientes diagnosticados con periodontitis refractaria presentan enterobacterias, señalándose también, que son menos susceptible a la clorhexidina, comparado con la mayoría de microorganismos presentes en la boca (14) Muchas enterobacterias tienen la capacidad para colonizar y proliferar en la cavidad bucal, actuando como cofactores en las formas destructivas de la enfermedad periodontal $(6,12,42)$.

\section{ASPECTOS EPIDEMIOLÓGICOS}

La prevalencia de las enterobacterias varía entre diferentes regiones del mundo (11-18). En Suecia se reportó la presencia de entéricos en $34,9 \%$ de las muestras siendo las especies más representativas Enterobacter cloacae y Klebsiella oxytoca (40). Similares resultados fueron encontrados, en adultos estadounidenses con periodontitis avanzada. Allí, la presencia de enterobacterias especialmente Enterobacter cloacae, Enterobacter agglomerans, Proteus mirabilis, Klebsiella pneumoniae y Klebsiella oxytoca, fue del $28 \%$ (41). En estudios multicéntricos realizados en Colombia, Chile y España se reportaron prevalencias del $36 \%$ y $17,6 \%$ en Colombia y Chile respectivamente, mientras que en España no se encontraron entéricos $(8,17,18)$. Las especies más frecuentemente encontradas en el estudio realizado en Colombia fueron la Klebsiella pneumoniae $y$ el Enterobacter cloacae. Estos hallazgos confirman que los microorganismos no residentes usualmente constituyen menos del $1 \%$ de la cantidad total viable en una muestra subgingival. En una población Rumana con periodontitis, se reportó la presencia de entéricos en el $61,1 \%$ de los pacientes (6). En una investigación realizada en Sudán se demostró la presencia de enterobacterias en el $92 \%$ de los pacientes pero contrario a un estudio en Noruega, en el cual ninguno 
de los pacientes fue positivo para estos microorganismos (12). En el Brasil se han observado prevalencias del $31,2 \%$ de entéricos en placa subgingival, encontradas principalmente en bolsas periodontales profundas (20). La frecuencia de enterobacterias en otros países son: República Dominicana 67\% (14) y China $57 \%$ (42). Es importante anotar que los estudios realizados en Colombia asocian la presencia de entéricos a condiciones demográficas y características culturales del país $(17,18)$. La tabla 2 presenta la prevalencia de entéricos en diferentes regiones del mundo.

La literatura ha sustentando la alta prevalencia de estos microorganismos en algunas regiones debidos a que la muestra fue transportada por más de 36 horas, por su presencia en alimentos, agua contaminada y también debido a mala higiene personal, además del uso incontrolado de agentes microbianos (12). Este último hecho es corroborado por una investigación en donde se demostró que la terapia sistémica con doxiciclina, puede originar un sobrecrecimiento de entéricos en la microflora de los pacientes con periodontitis crónica, debido a que el uso prolongado y repetitivo de tetraciclinas en pacientes puede facilitar una colonización subgingival permanente o prolongada, evitando la resolución de la infección periodontal (43).

La tabla 3 presenta las hipótesis posibles que respaldan la presencia de enterobacterias en la cavidad bucal.

\section{TRATAMIENTO}

El tratamiento en pacientes con periodontitis se realiza tradicionalmente mediante técnicas de raspado y

\section{TABLA 2.- PREVALENCIA DE ENTÉRICOS EN DIFERENTES REGIONES DEL MUNDO}

Suecia

Estados Unidos

Colombia

Chile.

Rumanía

Sudán

Brasil

República Dominicana

China
$34,9 \%$

$28,0 \%$

$36,0 \%$

$17,6 \%$

$61,1 \%$

$92,0 \%$

$31,2 \%$

$67,0 \%$

$57,0 \%$
(40)

(41)

$(8,17)$

(12)

(20)

(14)

(42)
TABLA 3.- HIPÓTESIS QUE RESPALDAN LA PRESENCIA DE ENTEROBACTERIAS EN LA CAVIDAD BUCAL

\begin{tabular}{|c|c|}
\hline 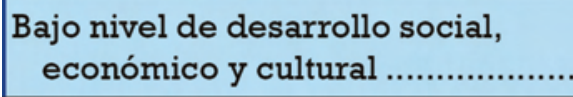 & (18) \\
\hline Contaminación mano-ano-boca ...... & $(14,15,18)$ \\
\hline 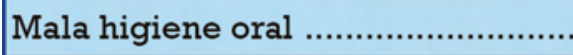 & $(14,15,16,41)$ \\
\hline $\begin{array}{l}\text { Ingestión de agua } 0 \text { alimentos } \\
\text { contaminados }\end{array}$ & $(14,15,41)$ \\
\hline 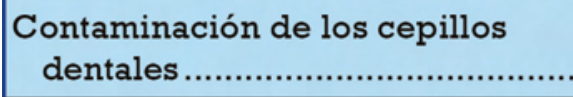 & $(40,41)$ \\
\hline Uso indiscriminado de antibióticos . & $(6,14,16,42,43$ \\
\hline 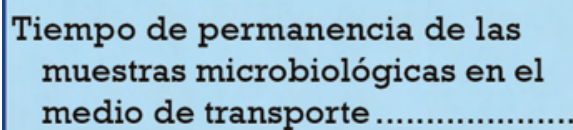 & $(6,15)$ \\
\hline
\end{tabular}

alisado radicular, sin embargo, diferentes investigaciones han demostrado que existen elementos que minimizan su éxito. Los factores que reducen la efectividad de la terapia mecánica convencional son: inmunosupresión, consumo de cigarrillo, malos hábitos de higiene oral, la extensión y la naturaleza de la pérdida de inserción, variaciones locales anatómicas (surcos, bifurcaciones, concavidades), el tipo de enfermedad periodontal y la composición microbiana de la placa subgingival (44). Slots (14), Listgarten (23) y Handal (24) encontraron que en pacientes tratados con terapia mecánica sola, no había mejoría en aquellas bolsas periodontales que presentaban enterobacterias, debido a que estas se encontraban principalmente en lesiones avanzadas.

Para mejorar los resultados de la terapia mecánica tradicional, se ha propuesto como tratamiento coadyuvante la utilización de antibióticos debido a la naturaleza infecciosa de la enfermedad periodontal. (20, 44-46). Los antibióticos administrados sistémicamente penetran los tejidos y las bolsas periodontales alcanzando microorganismos que son inaccesibles al raspado mecánico (44). La selección de antibióticos en la práctica clínica puede estar basada en análisis microbiológicos de muestras obtenidas de sitios afectados, pero este enfoque está limitado a casos de difícil tratamiento porque tales análisis pueden ser costosos, técnicamente complicados y requieren de laboratorios altamente estandarizados que reporten resultados con- 
fiables $(8,47)$. Otra dificultad de la selección de antibióticos, mediante pruebas de laboratorio, es que se ha demostrado mayor resistencia contra las bacterias presentes en la placa subgingival in vivo comparado con estudios in vitro $(48,49)$. Es así, como con regularidad, la selección de los antibióticos se realiza de manera empírica y se basa en los signos clínicos (44).

Los antibióticos que tradicionalmente se han utilizado como coadyuvantes a la terapia mecánica y que han demostrado efectividad clínica y microbiológica son: amoxicilina, amoxicilina/clavulánico tetraciclinas, metronidazol y azitromicina (5, 20, 44-47). Sin embargo, teniendo en cuenta que en algunas regiones se ha reportado la presencia de enterobacterias en placa subgingival en pacientes con periodontitis crónica, la respuesta al tratamiento puede ser deficiente. Los entéricos han mostrado resistencia a la amoxicilina, amoxicilina/clavulánico, a las tetraciclinas y al metronidazol en estudios realizados en Estados Unidos, Noruega, Brasil y Colombia (14, 16-18, 23, 24) Por otra parte, causado por el pobre control en la prescripción de medicamentos en algunos países, es posible que existan cepas resistentes a los antibióticos y sobrecrecimiento de patógenos en las bolsas periodontales (8). Esto ha ocasionado, que debido a la gran resistencia de los entéricos a la mayoría de antibióticos regularmente usados en la terapia periodontal, algunos autores hayan sugerido el uso de la ciprofloxacina, ya que ha demostrado resultados favorables en estudios in vitro $(14,16,18,23)$. Sin embargo, aunque la ciprofloxacina es un antibiótico de relativo amplio espectro, tiene actividad limitada contra los principales periodontopatógenos, lo cual implica utilizarla en combinación con otro antibiótico, siendo el de elección el metronidazol, debido a su sinergia con la ciprofloxacina y a su gran efectividad microbiológica contra los periodontopatógenos comunes $(14,16)$. La dosis recomendada es $500 \mathrm{mg}$ de ciprofloxacina dos veces al día durante 10 días y $250 \mathrm{mg}$ de metronidazol tres veces al día por 10 días $(14,50)$. También ha sido propuesta la combinación de ciprofloxacina con clindamicina y con antibióticos betalactámicos $(14,51,52)$ Aunque estas combinaciones de antibióticos han sido ampliamente sugeridas en la literatura odontológica, no se conocen ensayos clínicos aleatorizados publicados que demuestren su efectividad contra periodontopatógenos y entéricos presentes en placa subgingival de sujetos con periodontitis crónica.

Diferentes estudios han manifestado efectividad de la azitromicina contra enterobacterias (Klebsiella, Enterobacter, Salmonella, Shigella, E. Coli, Haemophilus I., S.
Aureus) causantes de enfermedades respiratorias, diarréicas, ginecobstétricas, urinarias, del tracto reproductivo y fiebre entérica (53-57), pero no se tiene conocimiento de estudios experimentales sobre susceptibilidad y resistencia antibiótica de la azitromicina a entéricos aislados de placa subgingival de pacientes con periodontitis crónica.

En la medida que las investigaciones han avanzado en el estudio de la medicina periodontal, la periodontitis ha sido considerada como una infección crónica de impacto sistémico, indicando que los protocolos de manejo en pacientes con riesgo cardiovascular y prenatal, incorporen la terapia periodontal. Sin embargo, las diferencias encontradas entre los estudios en países desarrollados y en vía de desarrollo, indican la necesidad de evaluar el efecto del tratamiento, en especial en aquellos pacientes con sobreinfección por enterobacterias, para de esta forma establecer el efecto de la terapia básica sobre la respuesta inflamatoria a nivel local y a nivel sistémico. Lo anterior implica la realización de estudios analíticos que indiquen los protocolos necesarios para tratar racionalmente los pacientes con periodontitis, basados en la mejor evidencia científica disponible.

\section{BIBLIOGRAFÍA}

1. Albandar JM, Epidemiology and risk factors of periodontal diseases. Dent Clin North Am 2005; 49:517-32.

2. Armitage, G. Development of a Classification System for Periodontal Diseases and Conditions. Ann. Periodontol 1999; 4:1-6.

3. Organización Mundial de la Salud. World Health Organization. Oral health surveys. Basic methods. Geneva (Switzerland):World Health Organization; 1997.

4. Socransky SS, Haffajje AD. Evidence of bacterial etiology: a historical perspective. Periodontol 2000 1994; 5:134-44.

5. Van Winkelhoff, Herrera D, Oteo A, Sanz M. Antimicrobial profiles of periodontal pathogens isolated from periodontitis patients in the Netherlands and Spain. J Clin Periodontol 2005;32:893-98.

6. Ali RW, Velcescu C, Jivanescu MC. Prevalence of 6 putative periodontal pathogens in subgingival plaque samples from Romanian adult periodontitis patients. J Clin Periodontol 1996; 23:133-9. 
7. Socransky SS, Haffajje AD, Cugini M.A, Smith CM, Kent RL Jr. Microbial complexes in subgingival plaque.J Clin Periodontol 1998;25:134-44.

8. Herrera D, Contreras A, Gamonal J, Otero A, Jaramillo A, Silva N, Sanz M, Botero JE, León R. Subgingival microbial profiles in chronic periodontitis patients from Chile, Colombia and Spain. J Clin Periodontol 2008;35:106-13.

9. Sanz M, van Winkelhoff AJ, Herrera D, Dellemijn-Kippuw $\mathrm{N}$, Simon R, Winkel E. Differences in the composition of the subgingival microbiota of two periodontitis populations of different geographic location. A comparison between Spain and The Netherlands. Eur J Dent Sci 2000;108: 383-92.

10. Sanz M, Lau L, Herrera D, Morillo J M, Silva A. Methods of detection of Actinobacillus actinomycetemcomitans, Porphyromonas gingivalis and Tannerella forsythensis in periodontal microbiology, with special emphasis on advanced molecular techniques: a review.J Clin Periodontol 2004;31:1034-47.

11. Umeda M, Chen C, Bakker I, Contreras A, Morrison J. L, Slots, J. Risk indicators for harboring periodontal pathogens. J Periodontol 1998;69:1111-18.

12. Haffajje $A D$, Borgen $A$, Hasturk H, Feres M, López NJ, Socransky SS. Subgingival microbiota of chronic periodontitis subjects from different geographic locations. J Clin Periodontol 2004;31:996-1002.

13. Lopez N J, Socransky S. S., Da S I, Japlit M R, Haffajee A D. Subgingival microbiota of chilean patients with chronic periodontitis. J Periodontol 2004;75:717-25.

14. Slots J, Feik D, Rams TE. Prevalence and antimicrobial susceptibility of Enterobacteriaceae, Pseudomonadaceae and Acinetobacter in human periodontitis. Oral Microbiol Inmunol 1990;5:149-54.

15. Ali RW, Bakken V, Nilsen R, Skaug N. Comparative detection frecuency of six putative periodontal pathogens in Sudanese and Norwegian adult periodontitis patients. J Periodontol 1994; 65:1046-52.

16. Barbosa FCB, Mayer MPA, Saba-Chuifi E, Cai S. Subgingival occurrence and antimicrobial susceptibility of enteric rods and pseudomonads from Brazilian periodontitis patients. Oral Microbiol Immunol. 2001;16:306-10.

17. Botero JE, Arce RM, Escudero M, Betancourth M, Jaramillo, A, Contreras A l. Ocurrence of periodontopatic and superinfecting bacteria in chronic an aggressive periodontitis subject in a Colombia population. J Periodontol. 2007;78:696-704.

18. Lafaurie GI, Contreras A, Baron A, Botero J, MayorgaFayad I, Jaramillo A, et al. Demographic, clinical, and microbial aspects of chronic and aggressive periodontitis in Colombia: a multicenter study.J Periodontol 2007: 78:629-39.

19. Souto R, Colombo AP. Prevalence of Enterococcus faecalis in subgingival biofilm and saliva of subjects with chronic periodontal infection. Arch Oral Biol. 2008; 53:155-60.

20. Colombo AP, Palmier R, Torres MC, Rosalém W., Mendes MC, Souto R, Uzeda M. Effects of Non-Surgical Mechanical Therapy on the Subgingival Microbiota of Brazilians With Untreated Chronic Periodontitis: 9Month Results. J Periodontol 2005:76:778-84.

21. Hughes, F J, Syed M, Koshy B, Marinho V, Bostanci N, McKay I J et al. Prognostic factors in the treatment of generalizad aggressive periodontitis: I. Clinical features and initial outcome. J Clin Periodontol 2006;33:663-70.

22. Apatzidou DA, Riggio MP, Kinane DF. Quadrant root planning versus same-day full-mouth root planing. II Microbiological findings. J Clin Periodontol 2004;31:141-8.

23. Listgarten MA, Lai CH, Young V. Microbial composition and pattern of antibiotic resistance in subgingival microbial samples from patients with refractory periodontitis. J Periodontol 1993;64:155-61.

24. Handal T, Olsen I, Walker CB, Caugant DA. Betalactamase production and antimicrobial susceptibility of subgingival bacteria from refractory periodontitis. Oral Microbiol Immunol. 2004;19:303-8.

25. Seymour GJ, Ford PJ, Cullinan MP, Leishman S, Yamazakiy K. Relationship between periodontal infections and systemic disease. Clin Microbiol Infect 2007; 13 Suppl 4:3-10.

26. Santos-Pereira SA, Giraldo PC, Saba-Chujfi E, Amaral RLG, Morais SS, Fachini AM, Goncalves AKS: Chronic periodontitis and pre-term labour in Brazilian pregnant women: an association to be analysed. J Clin Periodontol 2007;34:208-13.

27. Aguado JM, Lumbresras C. Infecciones por enterobacterias. Medicine 1998;7:3622-28. 
28. Eisenstein BI. Diseases caused by Gram-negative enteric bacilli. En: Isselbacher KJ, Braunwald E, Wilson JD, Petersdorf RG, Martin JB, Fauci AJ, Root RK. eds. Harrison's Principles of Internal Medicine (13 th ed.). New York: Mc Graw-Hill 1994; 661-9.

29. Ogawa T, Uchida H. Differential induction of IL-1 beta and IL-6 production by the nontoxic lipid A from Porphyromonas gingivalis in comparison with synthetic Escherichia coli lipid $A$ in human peripheral blood mononuclear cells FEMS. Immunol Med Microbiol. 1996;14:1-13.

30. Pasare $\mathbf{C}$ and Medzhitov R. Toll-Dependent Control Mechanisms of CD4 T Cell Activation. Immunology 2004;2:733-41.

31. Hamada S, Koga T. Nishihana T. et al. Characterization and immunologic activities of lipopolysacharides from periodontal bacteria. Adv Den Res 1988; 2:284.

32. Agarwal S, Piesco NP. Differential Expression of IL-1 $\beta$, TNF- $\alpha$, IL-6, and IL-8 in Human Monocytes in Response to Lipopolysaccharides from different Microbes. J Dent Res1995;74:1057-65.

33. Abul K. Abbas AH, Lichtman JS. Inmunologia celular y molecular. $2^{a}$ edición. Interamericana. Mc Graw-Hill. 1995.

34. Medzhitov R, Preston H P, Janeway C A. Human homologue of the Drosophila Toll protein signals activation of adaptive immunity. Nature 1997;24:394-7.

35. Tapping RI, Akashi S, Miyake K, Godowski PJ, Tobias PS. Toll-like receptor 4 , but not toll-like receptor 2 , is a signaling receptor for Escherichia and Salmonella lipopolysaccharides. J Immunol 2000; 165:5780-7.

36. Shapiro RA, Cunningham MD, Ratcliffe K, Seachord C, Blake J, Bajorath J et al. Identification of CD14 residues involved in specific lipopolysaccharide recognition. Infect Immun 1997;65:293-7.

37. Bainbridge BW, Page RC, Darveau RP. Serum antibodies to Porphyromonas gingivalis block the prostaglandin E2 response to lipopolysaccharide by mononuclear cells. Infect Immun 1997;65:4801-5.

38. Roberts FA, Richardson GJ, Michalek SM. Effects of Porphyromonas gingivalis and Escherichia coli lipopolysacharides on mononuclear phagocytes. Infect Immun 1997;65:3248-54.

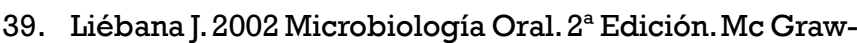
Hill. Interamericana. España.

40. Goldberg S, Cardash H, Browning H. Isolation of enterobacteriaceae from the mouth and potential association with malodor. J Dent Rest 1997; 76:1770-75.

41. Slots J, Lisgarten M. Bacteroides gingivalis, Bacteroides intermedius and Actinobacillus actinomycetemcomitans in human periodontal diseases. J Clin Periodontol 1988; 15:85-93.

42. Sedgley CM, Samaranayake LP, Chan JC, Wei SH. A 4year longitudinal study of the oral prevalence of enteric gram-negative rods and yeats in Chinese children. Oral Microbiol Inmunol 1997;12:183-8.

43. Rams TE, Babalola OO, Slots J. Subgingival ocurrence of enteric rods, yeasts and saphylococci after systemic doxycycline therapy. Oral Microbiol Inmunol 1990; 5:166-68.

44. Bidault P, Chandad F, Grenier D. Systemic antibiotic therapy in the treatment of periodontitis. J Can Dent Assoc. 2007;73:515-20.

45. Haffajee AD, Patel M, Socransky SS. Microbiological changes associated with four different periodontal therapies for the treatment of chronic periodontitis. Oral Microbiol Immunol 2008:23:148-157.

46. Gomi y col. Drug concentration in inflamed periodontal tissues after sistematically administered azithromycin. J Periodontol 2007; 78: 918-23.

47. Slots J. Update on human cytomegalovirus in destructive periodontal disease.Oral Microbiol Immunol 2004;19:217-23.

48. Sedlacek MJ \& Walter C. Antibiotic resistance in an in vitro subgingival biofilm model. Oral Microbiol Immunol 2007;22:333-39.

49. Goncalves MO, Coutinho-Filho WP, Pimenta FP, Pereira GA, Pereira JA, Mattos-Guaraldi AL et al. Periodontal disease as reservoir for multi-resistant and hydrolytic enterobacterial species. Letters in Applied Microbiology 2007;44:488-94.

50. Pankuch GA, Jacobs MR, Appelbaum PC. Susceptibilities of 428 Gram-Positive and -Negative Anaerobic Bacteria to Bay y3118 Compared with Their Susceptibilities to Ciprofloxacin, Clindamycin, Metronidazole, 
Piperacillin, Piperacillin-Tazobactam, and Cefoxitin. Antimicrob Agents Chemothe 1993;37:1649-54.

51. Esposito S, Gupta A, Thadepalli H. In vitro synergy of ciprofloxacin and three other antibiotics against Bacteroides fragilis. Drugs Exp Clin Res 1987;13:489-92.

52. Whitling JL, Cheng N, Chow AW. Interactions of ciprofloxacin with clindamicyn, metronidazole, cefoxitin, cefotaxime, and mezlocillin against grampositive and gram-negative anaerobic bacteria. Antimicrob Agents Chemother 1986;17:679-80.

53. Marcos LA, Dupont HL. Advances in defining etiology and new therapeutic approaches in acute diarrhea. Journal of Infection 2007;55:385-93.

54. Dipika S, Shanta Dutta BL, Sarkar B, Manna MK, Bhattacharya K.K, Datta A et al. Occurrence, significance \& molecular epidemiology of cholera outbreaks in West Bengal. Indian J Med Res 2007;772-76.
55. Capoor MR, Rawat D, Nair D, Hasan AS, Deb M, Aggarwal $P$ et al. In vitro activity of azithromycin, newer quinolones and cephalosporins in ciprofloxacin-resistant Salmonella causing enteric fever. J Med Microbiol 2007;56:1490-94.

56. Aboyeji AP, Abdul AF, Ijaiya MA, Nwabuisi C, Ologe MO. The bacteriology of pre-labour rupture of membranes in a Nigerian teaching hospital.J of Obstet and Gynaecol 2005;25:761-64.

57. Gupta DN, Mondal SK, Sarkar BL, Mukherjee S, Bhattacharya SK. An el tor cholera outbreak amongst tribal population in Tripura. J Commun Dis. 2004; 36:271-6.

\section{CORRESPONDENCIA}

Carlos Martín Ardila Medina

Carrera 47 No. 20 sur 46 Envigado Antioquia 57(4) 3348122

E-mail:cmartin@odontologia.udea.edu.co 